\title{
Abnormal Working Hours: Effect of Rapid Releases and Implications to Work Content
}

\author{
Maëlick Claes*, Mika Mäntylä*, Miikka Kuutila* and Bram Adams ${ }^{\dagger}$ \\ * M3S, ITEE, University of Oulu, Finland \\ Email: firstname.lastname@oulu.fi \\ $\dagger$ MCIS, Polytechnique Montreal, Canada \\ Email: bram.adams@polymtl.ca
}

\begin{abstract}
-
During the past years, overload at work leading to psychological diseases, such as burnouts, have drawn more public attention. This paper is a preliminary step toward an analysis of the work patterns and possible indicators of overload and time pressure on software developers with mining software repositories approach. We explore the working pattern of developers in the context of Mozilla Firefox, a large and long-lived open source project. To that end we investigate the impact of the move from traditional to rapid release cycle on work pattern. Moreover we compare Mozilla Firefox work pattern with another Mozilla product, Firefox OS, which has a different release cycle than Firefox. We find that both projects exhibit healthy working patterns, i.e. lower activity during the weekends and outside of office hours. Firefox experiences proportionally more activity on weekends than Firefox OS (Cohen's $d=0.94$ ). We find that switching to rapid releases has reduced weekend work (Cohen's $d=1.43$ ) and working during the night (Cohen's $d=0.45$ ). This result holds even when we limit the analyzes on the hired resources, i.e. considering only individuals with Mozilla foundation email address, although, the effect sizes are smaller for weekends (Cohen's $d=0.64$ ) and nights (Cohen's $d=0.23$ ). Moreover, we use dissimilarity word clouds and find that work during the weekend is more technical while work during the week expresses more positive sentiment with words like "good" and "nice". Our results suggest that moving to rapid releases have positive impact on the work health and work-life-balance of software engineers. However, caution is needed as our results are based on a limited set of quantitative data from a single organization.
\end{abstract}

\section{INTRODUCTION}

Factors relating to occupational health and wellbeing are seldom addressed in the software engineering body of knowledge. This is surprising considering the productivity in software development is largely related to peopleware, and large productivity differences have been documented [1]. Furthermore, replacing key individuals with in-depth knowledge of the product with new employees may take years as the new employees need ample time to gain knowledge about the product. As the matters of occupational health and wellbeing can reduce staff turnover, sick days and decrease mortality among software engineers, addressing them with mining software repositories becomes an important objective.

Long working hours have been associated with depressive state, anxiety, sleep condition, and coronary heart disease [2]. Importance of weekend recovery has been showed to affect weekly job performance personal initiative, organizational citizenship behaviour, and lower perceived effort [3]. Another study showed that psychological detachment during off-work time reduced emotional exhaustion caused by high job demands and helped to maintain work engagement [4]. Given the importance of the off-work time in employee recovery we investigate software developers work patterns from large open source project Mozilla Firefox with many hired resources. We are particularly interested in work performed outside of office hours as such working hours can acts as a proxy for job related stress and time pressure conditions and suggest non-sufficient detachment from work.

Mozilla Firefox is a web browser with widespread and long lived history. Firefox makes an interesting case study as it is supported by the Mozilla Foundation, that pay software engineers to develop it. Moreover, it moved from a slow to a fast release cycle in 2011. Until the release of Firefox 4.0 in March 2011, new major versions were released not more than once in a year and parallel versions kept being maintained for security updates. Following Firefox 4.0, a new major version is to be released every 6 weeks and supersede its previous version. This change from a slow to a fast release cycle has a potential impact on work patterns.

We investigate the following research questions:

RQ1 What is the developers' usual pattern of work activity? We look when developers are more likely to be active in both Mozilla Firefox and Firefox OS.

RQ2 Is there a difference in the developers' work pattern when switching to rapid releases? We compare the difference in work pattern in Mozilla Firefox before and after switching to rapid releases.

RQ3 Do developers show a different behavior outside office hours? Using basic text mining techniques we analyze the vocabulary more likely to be used outside and during office hours.

This paper is structured as follow. In Section II we list related work. In Section III we give details on how we extracted data to answer our research questions that we answer in Section IV. We then present the threats to validity that can impact our results in Section V and conclude in Section VI.

\section{RELATED WORK}

Sall et al. [5] studied weekend work activity patterns in San Francisco Bay Area using surveys. Results indicate that a host of variables affect the likeliness of working during the 
weekend. They found that gender, race, type of work and income affect the work pattern. Individuals are more likely to work out of home during weekends in winter season than other seasons.

Wang et al. [6] examined work patterns of scientists by looking at the amount of scientific papers being downloaded in different days. Scientists work for $60-70 \%$ as much during the weekend as during the week. Time worked during weekends differs by country: scientists work proportionally more during weekends in China than in USA and Germany.

Binnewies et al. [3] investigated the importance of recovery during weekend and its implications on work performance. Data from surveys indicate that experiences of psychological detachment, relaxation and mastery during weekend were positively correlated with of being recovered beginning the working week, which in turn was positively related to self reported work performance.

McKee [7] collected the findings of increased mortality rate in hospitals during the weekend, with explanations ranging from more seriously sick patients to less experiences staff. At extreme, the weekend effect has been observed to be $44 \%$ higher odds of mortality in Friday compared to Monday [8]. However, multiple sources state conflicting evidence on the source of this effect [7], [8], [9].

In software context, it has been observed that commits made between mid-night and 4 AM contain more bugs, while commits made between $7 \mathrm{AM}$ and noon contain the least [10]. However, sentiment analysis of commit messages finds more negative emotion on Mondays compared to other days [11].

Khomh et al. [12] studied the impact of Firefox's fast release cycle on post-release bugs. They found that not only the new release cycle didn't increase the number of bugs, but bugs are also fixed faster. The real challenges faced by the switch of release cycle was related to the need of automating the release engineering process.

\section{DATA EXTRACTION}

According to Mozilla's guidelines, every change must be reviewed by its component owner. For that to happen, an entry must be added in the bug tracker regardless of the type of change (bug/security fix, performance enhancements, new feature, etc). We extracted bug comments from Mozilla's Bugzilla repository ${ }^{1}$ using the GrimoireLab tools ${ }^{2}$. We filtered the bugs to only keep the ones related to the following products: Firefox, Core and Firefox OS.

However Bugzilla's data lacks time zone information. In order to be able to identify when people are working, we extracted timezone information from Mozilla unified Mercurial repository ${ }^{3}$. We matched contributors to the bug tracker with contributors with the source code repository using e-mail addresses. This allows us to focus on bug tracker contributors who have also contributed code, and thus can be considered as developers. While we were able to associate a timezone to only

\footnotetext{
${ }^{1}$ http://bugzilla.mozilla.com

${ }^{2}$ https://grimoirelab.github.io/

${ }^{3}$ https://hg.mozilla.org/mozilla-unified
}

2637 out of $145691(1.8 \%)$ of the bug tracker contributors, 5.86 millions of comments out 11.11 millions $(53 \%)$ were authored by them.

Each bug can have files, which are often patches, attached to it. Because we want to focus on development activity, we will only consider comments that were related to patch submission and code review. These comments can easily be identified as the comments starting with "Created attachment" and "Comment on attachment". However we ignore those starting lines when analyzing the text of the comments. We also ignored comments that were posted by e-mail addresses known to be used by bots.

Analyses comparing Firefox and Firefox OS were made using comments written between 2012-12-21 and 2016-0103 . These dates correspond to the feature complete date of Firefox OS 1.0 and code complete release date of Firefox OS 2.5. In other words, we limit our analysis to the time when professional development of Firefox OS took place. Firefox browser development was professional during the entire time.

\section{EMPIRICAL ANALYSIS}

\section{A. What is the developers' usual work pattern?}

First we computed the number of comments related to attached files for each day. Fig. 1 shows that comments are not evenly distributed over the days of the week. Indeed fewer comments are posted during the weekend.

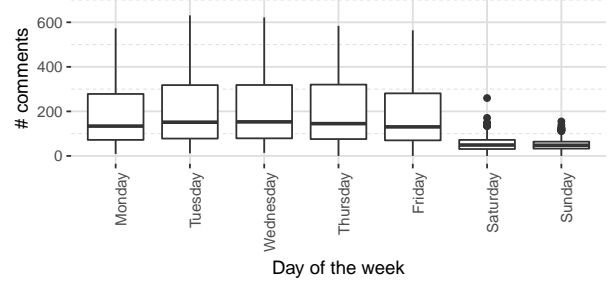

Fig. 1. Distribution of the number of comments for Firefox depending on the day of the week.

Similarly, we computed the number of comments for each hour of the day. Fig. 2 shows that most activity occurs between 9 am and $7 \mathrm{pm}$. Overall the usual work pattern of Firefox developers follow regular office hours with low activity during the night and during the weekends.

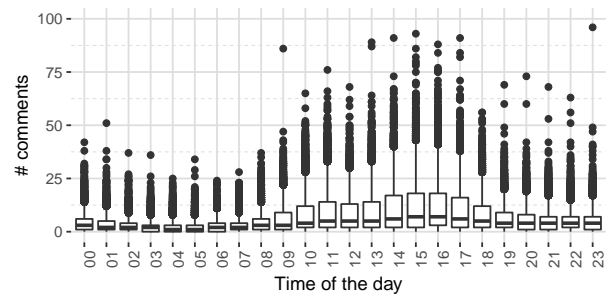

Fig. 2. Distribution of the number of comments for Firefox depending on the time of the day.

We compare Firefox work pattern with another Mozilla project: Firefox OS. Fig. 3 and Fig. 4 shows that Firefox OS 
developers follow the same work pattern. Based from these observations, we identified the period from 8 am to $7 \mathrm{pm}$ as the day and from $8 \mathrm{pm}$ to $7 \mathrm{am}$ as the night. For both systems we computed the proportion of daily comments made during the night, and the proportion of comments made during a weekend day compared to a weekday. We observed that relative activity in Firefox OS is significantly lower during weekends than for Firefox. While we both observe a statistically significant difference between projects for both weekend and night work, the effect size of the weekend work is large (Mann-Whitney $\mathrm{U}$ test $p$-value $<0.001$, Cohen's $d=0.94$, Cliff's $\delta=0.77$ ) and neglible for night work (Mann-Whitney $\mathrm{U}$ test $p$-value $<0.01$, Cohen's $d=0.06$, Cliff's $\delta=0.06$ ). On average for Firefox, a weekend day receives $24 \%$ of the comments a weekday receives, while for Firefox OS a weekend day receives $12.2 \%$ of the comments of a weekday.

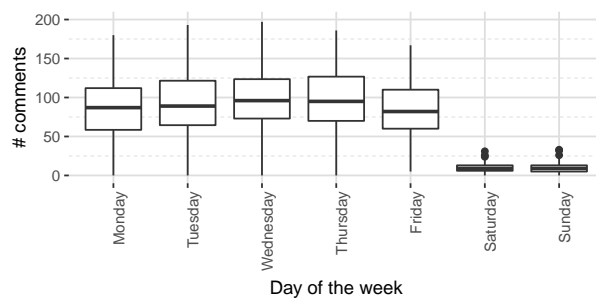

Fig. 3. Distributions of the number of comments for Firefox OS by day of the week.

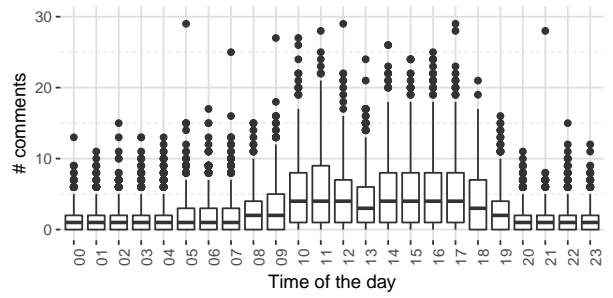

Fig. 4. Distributions of the number of comments for Firefox OS by time of the day.

This means that even though Firefox shows a rather healthy work pattern, differences can exist between projects. One possible explanation is the difference in contributor profiles. Open source projects attract hobbyist developers who might have a regular paid job and contribute to the project during their free time. Differences in the amount of such developers can potentially explain differences in work pattern. While we observe lower weekend and night activity when focusing solely on contributors using a Mozilla e-mail address, there is still an important difference for the weekend activity between Firefox and Firefox OS (Mann-Whitney U test $p$-value $<0.001$, Cohen's $d=0.53$, Cliff's $\delta=0.6$ ).

\section{$B$. Is there a difference in the developers' work pattern when switching to rapid releases?}

Fig. 5 shows both the evolution over time of the number of weekly comments posted during weekends and weekdays, and the evolution of those posted during the night and during the day. While the number of comments posted during office hours has increased significantly since 2012, the same trend cannot be observed for the night and weekend comments. We compared the distribution of the percentage of comments during weekends and nights before and after moving to rapid releases. There is a statistically significant decrease in the relative amount of comments made outside office hours. However this decrease is more important for weekend work (Mann-Whitney U test $p$-value $<0.001$, Cohen's $d=1.43$, Cliff's $\delta=0.78$ ) than night work (Mann-Whitney U test $p$-value $<0.001$, Cohen's $d=0.45$, Cliff's $\delta=0.33$ ). On average since the release of Firefox 4, a weekend day receives $24.4 \%$ of the comments a weekday receives, while before Firefox 4, a weekend day used to receive $48.6 \%$ of the comments of a weekday.

Again, the difference might be explained by an increase in the number of developers paid by Mozilla. Indeed $42.5 \%$ of all comments since the fast release cycle have been written by people using a Mozilla e-mail address, while only $16.7 \%$ of comments were before. However, even when taking into accounts only comments posted with a Mozilla e-mail address, we still observe a large decrease in the activity during weekends (Mann-Whitney U test $p$-value $<0.001$, Cohen's $d=0.64$, Cliff's $\delta=0.52$ ) but a small to negligible one during the nights (Mann-Whitney $\mathrm{U}$ test $p$-value $<0.01$, Cohen's $d=0.23$, Cliff's $\delta=0.05$ ). For comments posted with a Mozilla e-mail addresses, on average since the release of Firefox 4, a weekend day receives $17.2 \%$ of the comments a weekday receives, while before Firefox 4, a weekend day used to receive $52.4 \%$ of the comments of a weekday.

\section{Do developers show a different behavior outside office hours?}

In order to determine if there is a difference in the type of comments developers write outside office hours, we computed the frequency of appearance of the words from all comments. Using the wordcloud $\mathrm{R}$ package ${ }^{4}$ we computed a comparison wordcloud depicted in Fig. 6. It highlights words that have a highest probability to appear in a comment posted during a weekend or during a weekday.

First many of the most likely words to appear in a weekend comment are technical words. These include words like testcase, assertion, int, bool, file, unsigned, fix, debug, bytes, error or crash. On the other hand, weekday comments are more likely to have non technical words like add, since, instead, will, use, see, looks, need, think, maybe or can. Moreover, we also see positive or polite words like good, please, thanks and nice. This hints that developers tend to talk more during weekdays than during weekends where they focus more on technical aspects. The differences between the day and night are similar but not as strong (figure ommited due to space restrictions).

Then words related to reviews are also more likely during the weekdays. In particular, nit, a shortcut of nitpicking, is

\footnotetext{
${ }^{4}$ https://cran.r-project.org/web/packages/wordcloud/index.html
} 

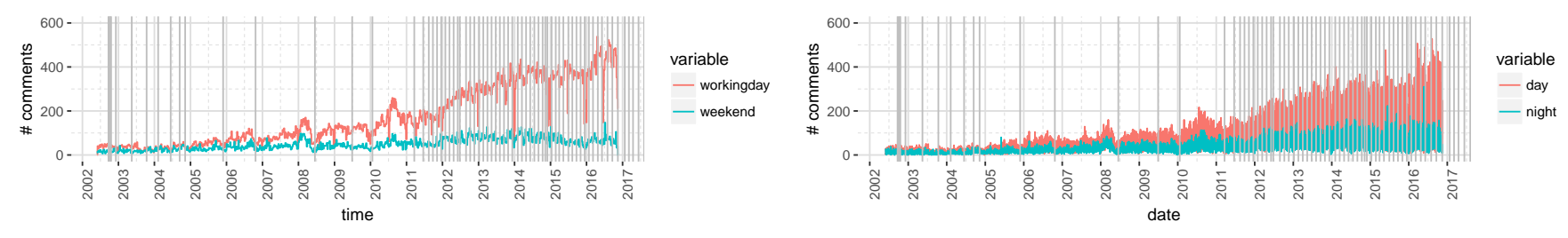

Fig. 5. Evolution of the number of comments for Firefox. Vertical gray lines represent major Firefox releases.

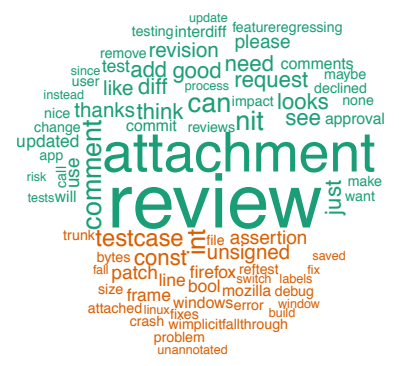

Fig. 6. Comparison wordcloud of comments posted during weekends and weekdays. Words on the bottom, in orange, have a higher probability to appear in comments during weekends than words on the top, in green, which have a higher probability to appear in comments during weekdays.

used in code review by the Mozilla community to indicate less import comments to take into account. This might indicate that developers tend to be more direct and focus on what's the most important when working outside the regular work pattern.

\section{Threats To VALIDITy}

Our analysis depends on data extracted from repositories available from online sources. Errors or incompleteness in these data sources may impact the result of our analysis. In particular we relied on time zone information available from Mozilla's Mercurial source code repository. The time zone from this repository might not be accurate to the developer's actual position. Moreover, we only used e-mail addresses to match identities from the Mercurial repository with the authors of comments in the Bugzilla repository. We are aware of some developers using a different e-mail address in each repository.

We limited our study to a single organization and the results obtained might be specific to that organization culture and habits of its developers. Similarly this can also explain similarities in work pattern between Firefox and Firefox OS.

In order to identify developers hired by Mozilla, we used the domain name of the e-mail address used as a proxy. We are aware of a few major Mozilla developers who don't use a Mozilla e-mail address. Finally, the scripts that we have developed for our empirical analysis may still contain some bugs, and the obtained results may be biased by some simplifying assumptions we have made during our analysis.

\section{CONCLUSION AND FUTURE WORK}

In this paper we investigated software developers' work patterns and possible indicators of overload and time pressure with mining software repositories approach in the context of Mozilla Firefox. We find that activity is lower outside office hours. We find a rather important difference in the activity during weekend when comparing Firefox with Firefox OS. We also find that the tendency to work outside office hours, in particular during weekends, has reduced over the years. While the differences can be explained by the amount of developers hired by Mozilla, we find that the findings still hold when limiting our analysis to Mozilla developers.

A common assumption of faster release cycle could propose that: as the number of deadlines increases, this increases time pressure related to deadlines, which increases abnormal work patterns, i.e., working during nights and weekends. However, it appears that the opposite is true as switching to rapid releases has reduced the amount of work performed outside office hours. Studies from psychology partially support this: it was found that more frequent deadlines reduces the likelihood of being late from the final deadline [13]. Thus, it seems that rapid release cycles have a positive effect on reducing work outside office hours and lead to increased occupational health.

Moreover, we found that comments posted on weekends contained more often technical terms than those posted on weekdays. On the other hand, comments posted on weekdays usually contained more positive or polite words, such as nice, please or thanks. This hints that developers tend to be more direct in their comments and focus only on the technical aspect instead of formalities outside office hours.

As future work, we intend to further study the impact of time pressure and work patterns on software developers. We aim to compare multiple projects to see whether we could empirically draw normative recommendations of outside office work in software projects. We will also use additional data sources, such as commits and chat logs, in order to make our set of timestamp activity more complete, and conduct interviews with development teams. Measuring the level of detachment from work that is critical to recovery, should be studied and similarly normative recommendation from empirical data should be drawn. We also intend to study the impact of policies and guidelines put in place by project managers, such as a fast release cycle, on developers' activity and health. We also want to investigate further the causes of outside office hours work by focusing on periods with unusual high activity during the night or weekends.

\section{ACKNOWLEDGMENTS}

The authors have been supported by Academy of Finland grant 298020. 


\section{REFERENCES}

[1] S. McConnell, "What does 10x mean? measuring variations in programmer productivity," Making Software, O'Reilly, vol. 16, 2011.

[2] A. Bannai and A. Tamakoshi, "The association between long working hours and health: a systematic review of epidemiological evidence," Scandinavian journal of work, environment \& health, vol. 40, no. 1 , pp. 5-18, 2014.

[3] C. Binnewies, S. Sonnentag, and E. J. Mojza, "Recovery during the weekend and fluctuations in weekly job performance: a week-level study examining intra-individual relationships," Journal of Occupational and Organizational Psychology, vol. 83, no. 2, pp. 419-441, 2010.

[4] S. Sonnentag, C. Binnewies, and E. J. Mojza, "Staying well and engaged when demands are high: the role of psychological detachment." Journal of Applied Psychology, vol. 95, no. 5, p. 965, 2010.

[5] E. A. Sall and C. R. Bhat, "An analysis of weekend work activity patterns in the san francisco bay area," Transportation, vol. 34, no. 2, pp. 161-175, 2007. [Online]. Available: http: //dx.doi.org/10.1007/s11116-006-0008-2

[6] X. Wang, S. Xu, L. Peng, Z. Wang, C. Wang, C. Zhang, and X. Wang, "Exploring scientists' working timetable: Do scientists often work overtime?" CoRR, vol. abs/1208.2686, 2012. [Online]. Available: http://arxiv.org/abs/1208.2686

[7] M. McKee, "The weekend effect: now you see it, now you don't," $B M J$, vol. 353, 2016. [Online]. Available: http://www.bmj.com/content/ 353/bmj.i2750
[8] P. Aylin, "Making sense of the evidence for the "weekend effect"," $B M J$, vol. 351, 2015. [Online]. Available: http://www.bmj.com/content/ $351 / \mathrm{bmj} . \mathrm{h} 4652$

[9] B. Alexander, M. Dijst, and D. Ettema, "Working from 9 to 6 ? an analysis of in-home and out-of-home working schedules," Transportation, vol. 37 , no. 3 , pp. 505-523, 2010. [Online]. Available: http://dx.doi.org/10.1007/s11116-009-9257-1

[10] J. Eyolfson, L. Tan, and P. Lam, "Do time of day and developer experience affect commit bugginess?" in Proceedings of the 8th Working Conference on Mining Software Repositories, ser. MSR '11. New York, NY, USA: ACM, 2011, pp. 153-162. [Online]. Available: http://doi.acm.org/10.1145/1985441.1985464

[11] E. Guzman, D. Azócar, and Y. Li, "Sentiment analysis of commit comments in github: An empirical study," in Proceedings of the 11th Working Conference on Mining Software Repositories, ser. MSR 2014. New York, NY, USA: ACM, 2014, pp. 352-355. [Online]. Available: http://doi.acm.org/10.1145/2597073.2597118

[12] F. Khomh, B. Adams, T. Dhaliwal, and Y. Zou, "Understanding the impact of rapid releases on software quality," Empirical Softw. Engg., vol. 20, no. 2, pp. 336-373, Apr. 2015. [Online]. Available: http://dx.doi.org/10.1007/s10664-014-9308-x

[13] D. Ariely and K. Wertenbroch, "Procrastination, deadlines, and performance: Self-control by precommitment," Psychological science, vol. 13 , no. 3, pp. 219-224, 2002. 